\title{
Efficacy of Plastic Reconstruction (Limberg Rhomboid Flap) in Management of Sacrococcygeal Pilonidal Sinus
}

\author{
Mohamed Aly Elhorbity \\ Department of General Surgery, Benha Teaching Hospital, General Organization for Teaching Hospitals and Institutes, Benha, Egypt \\ Email address: \\ mohamed_elhorbity@hotmail.com

\section{To cite this article:} \\ Mohamed Aly Elhorbity. Efficacy of Plastic Reconstruction (Limberg Rhomboid Flap) in Management of Sacrococcygeal Pilonidal Sinus. \\ Advances in Surgical Sciences. Vol. 6, No. 1, 2018, pp. 31-35. doi: 10.11648/j.ass.20180601.16
}

Received: May 22, 2018; Accepted: June 12, 2018; Published: July 4, 2018

\begin{abstract}
Sacrococcygeal pilonidal sinus disease is a common condition usually seen in young adult males. The definitive treatment of sacrococcygeal pilonidal sinus is a surgical excision of all sinus tracts. The surgical procedures range from simple excision with or without primary closure to complex flap reconstruction. However, no single operative intervention is superior to another based upon overall rate of healing, time away from work, and risk of recurrence. Between January 2014 and march 2017, a total of 30 patients (28 male and 2 female) aged between 17 and 40 years old complaining from sacrococcygeal pilonidal sinus, 10 cases are recurrent after previous operation ( 6 recurrent cases after excision and simple primary closure and the another 4 recurrent cases after excision and lay open the wound to heal with secondary intension). Another 12 cases diagnosed several months after drainage of previous surgical drainage of pilonidal abscess with persistent non healed sinus and the remaining 8 cases are chronic pilonidal sinus with no history of previous abscess or operations. All cases after proper investigation managed with Limberg rhomboid flap for wound closure after surgical excision of the sacrococcygeal pilonidal sinus. The mean operative time was ranged from 50 to 70 minutes (average 60 minutes). Most cases (25 patients) received spinal anesthesia and the remaining (5 patient) received general anesthesia according to their desire. All patients discharged home $24 \mathrm{~h}$ to 48 after the operation and only one recurrent case need admission again for reoperation within 6 months. No recorded cases of wound infection, or flap necrosis were observed. All patients returned to work from 2 to 4 weeks after the operation with minimal postoperative pain with no wound tension or irritability and all were satisfied. The present investigation was concluded that the sacrococcygeal pilonidal sinus is chronic disease and surgeons have been treating it by different modalities range from lay open technique to wound closure either simple or based on plastic flap reconstruction. Limberg rhomboid flap reconstruction after excision of sacrococcygeal pilonidal sinus is meticulous, safe, easy to be done, low operative time, low post-operative pain, low hospital stays, early return of the patient to work, suitable to primary and recurrent cases with low local recurrent and meet acceptance from the patient preoperative during discussion with the patient for writing the surgical consent and postoperative due to the previous benefits.
\end{abstract}

Keywords: Plastic Reconstruction, Limberg Rhomboid Flap, Sacrococcygeal Pilonidal Sinus

\section{Introduction}

Pilonidal sinus is a chronic intermittent disease, usually involving the sacrococcygeal area and it was first described by Hodges in 1880 [1]. The specific mechanism for the development of pilonidal disease is unclear, although the presence of hair and inflammation in the natal cleft are contributing factors [2].

The incidence of pilonidal disease is approximately 26 per 100,000 populations, with a mean age at presentation of 19 years for women and 21 years for men, with men being affected two to four times more often than women [2]. For more than hundred years, surgeons have been treating this disease by various treatment modalities, including simple incision and drainage, laying open, marsupialization, excision and primary closure, or rhomboid excision with Limberg flap procedure [3].

A primary closure is associated with faster wound healing (complete epithelialization) and a sooner return to work, but a delayed (open) closure is associated with a lower likelihood of pilonidal disease recurrence. This is best illustrated by a metaanalysis of 26 randomized trials including 2530 patients [4]. 
The rhomboid or Limberg flap is a rotational fasciocutaneous flap that permits primary off-midline closure of the wound and flattening of the gluteal cleft [5]. The reported recurrence rate ( 0 to 6 percent) and surgical infection rate ( 0 to 6 percent) are both low and compare favorably to those of simple midline closure in some studies [6].

There have been many studies reporting a recurrence rate of $7-42 \%$ following excision and primary closure; however, a recurrence rate of about $3 \%$ has been reported following Limberg flap repair [7].

\section{Aim of Work}

The aim of this study is to assess the efficacy of Limberg rhomboid flap as suitable technique for surgical management of primary and recurrent sacrococcygeal pilonidal sinus according to post-operative pain, short time of wound healing, minimal wound infection, early return to work and low recurrence rate.

\subsection{Patients and Methods}

Between January 2014 and March 2017, Limberg rhomboid flap procedure was successfully used in 30 cases complaining from of sacrococcygeal pilonidal sinus. Most of the patients were males ( 28 males and 2 females) and the mean age was ranged from 17 to 40 years. 10 cases are recurrent after previous operation (6 recurrent cases after excision and simple primary closure and another 4 recurrent cases after excision and lay open the wound to heal with secondary intension). Another 12 cases diagnosed several months after drainage of previous surgical drainage of pilonidal abscess with persistent non-healed sinus and the remaining 8 cases are chronic pilonidal sinus with no history of previous abscess or operations. All patients were informed about the purpose of the study with ethical aspects and a written consent was taken. During the study, duration of the symptoms, body mass index (BMI), past medical history, history of previous operations or abscess formation were recorded. Following the operation, type of anesthesia, duration of hospital stays, degree of post-operative pain using pain scoring scale, patient satisfaction, postoperative wound complications, duration return to normal activities and work, recurrent cases, length o follow up were recorded.

\subsection{Exclusion Criteria}

Exclusion of morbid obese (BMI more than $35 \mathrm{~kg} / \mathrm{m}^{2}$ ) patients, American Society of Anesthesiologists (ASA) group higher than III, diabetes, and severe allergy to local anesthetics or other medications were happen. All patients received intravenous third generation cephalosporins (cefotaxime) $1 \mathrm{gm}$ just before induction of anesthesia followed by $1 \mathrm{gm}$ each 12 hour for 24 hours postoperative. All patients were prescribed $75 \mathrm{mg}$ diclofenac intramuscular (IM) each 12 hour in the early 24 hour postoperative and additional dose of $50 \mathrm{mg}$ tramadol slow intravenous (IV) when needed. After hospital discharge all patient prescribed oral levofloxacin $500 \mathrm{mg}$, once daily for 10 days and oral Ibubrofen $400 \mathrm{mg}$ each 8 hour for one week in addition to $100 \mathrm{mg}$ tramadol intramuscular when needed.

\subsection{The Procedure}

Surgery is performed either in spinal (25 cases) or general ( 5 cases) anesthesia. Patient is placed in prone position with buttocks elevated and taped apart to open the natal cleft for wide exposure. After adequate shaving and skin preparation, area to be excised is carefully marked and flap lines are mapped on the skin.

The rhomboid incision (with each side equal in length), includes the sinus, is made down to the presacral fascia. The flap is constructed by extending the incision laterally down to the fascia of the gluteus maximus muscle. Complete hemostasis is done by the use of electrocautery. The flap is transposed to the rhomboid defect after excision of the sinus. The wound is closed after putting closed suction drain from separate stab wound, the subcutaneous layer sutured with interrupted vicryl 2 zero and the skin with interrupted monoaryl 3 zero.

Patients discharged home after 24 to $48 \mathrm{hrs}$ postoperative and the drain removed from 2 to 5 days postoperative. Sutures are removed from 10 to 14 days postoperative. Patients are advised to avoid prolonged sitting or exercise for two weeks. Hair removal either by hair clipper or by hair removal cream regularly each month is advised for at least 6 months. Patients are followed up in outpatient clinic monthly for 6 months.

Duration of hospital stay, postoperative wound complication, time return to normal activities and work, recurrent cases all were recorded. Postoperative degree of pain using pain scoring scale also was recorded.

\section{Results}

Thirty patients were operated by Limberg rhomboid flap reconstruction in this study. Most of the patients were male ( 28 male and 2 female) and the mean age was ranged from 17 to 40 age. All patients discharged home $24 \mathrm{~h}$ to 48 after the operation, with no cases of wound infection, no cases of flap necrosis, one case of wound seroma and one case of hematoma due to fall the patient on his back 2 weeks after the operation. The seroma case managed conservatively after local drainage under local anesthesia in outpatient clinic and the hematoma case not respond to conservative management after local drainage and resulted in local recurrence in the same case. One case of small wound japing in the upper end of the flap and another 2 cases of small wound japing in the lower lateral end of the flap were recorded which all completely healed after 4-6 weeks of conservative wound daily dressing after complete stitches removal.

Time of normal ambulation, walking without pain, no need for oral analgesic from 10 to 14 days and time to return to work and normal activities from 2 to 4 weeks were reported. Postoperative pain according to pain scoring scale was mild 
to moderate early post-operative period with good response to oral ibuprofen with no reported cases of chronic pain.

\section{Discussion}

Pilonidal disease was originally thought to be congenital due to abnormal skin in the gluteal cleft [8].

But recent theories consider it acquired rather than congenital [9]. Main causes for the formation of this sinus are hirsutism, sweating in the area, repeated maceration due to trauma, leading to breakage of the skin barrier, attracting hair inside which initiates a foreign body reaction leading to infection with abscess or sinus formation.

There are many surgical operations for treatment of sacrococcygeal pilonidal sinus varies from leave the wound open after excision of the pilonidal sinus tract to heal with secondary intention or primary closure of the wound with or without plastic flap reconstruction. The best surgical technique for sacrococcygeal pilonidal disease is still controversial [10].

Figure legends:

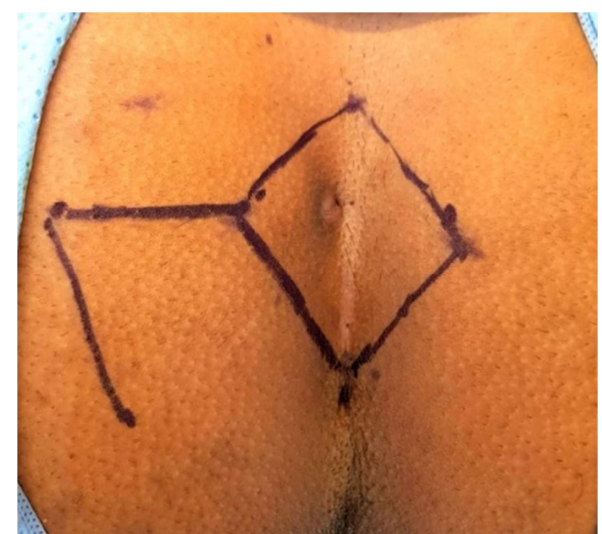

Figure 1. Showing the design of the rhomboid incision, in primary case includes the sinus.

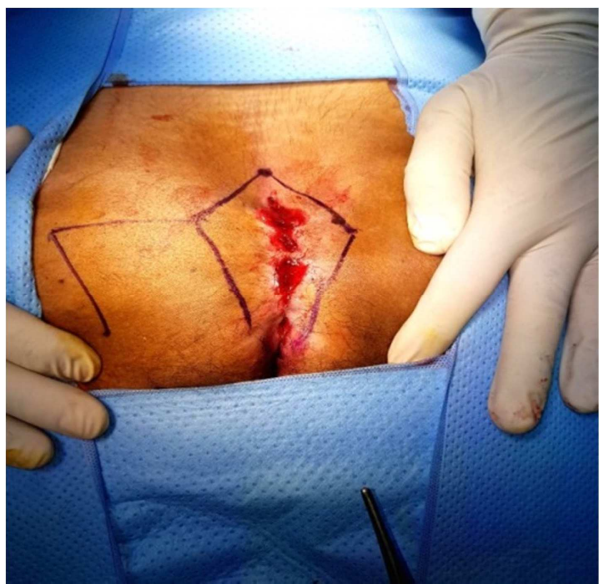

Figure 2. Showing the design of the rhomboid incision, in recurrent case includes the sinuses.

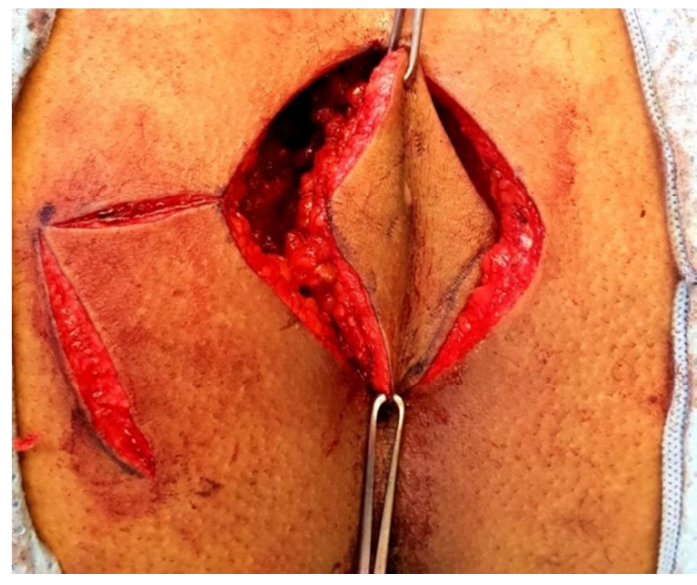

Figure 3. Showing the flap is constructed by extending the incision laterally down to the fascia of the gluteal maximus muscle.

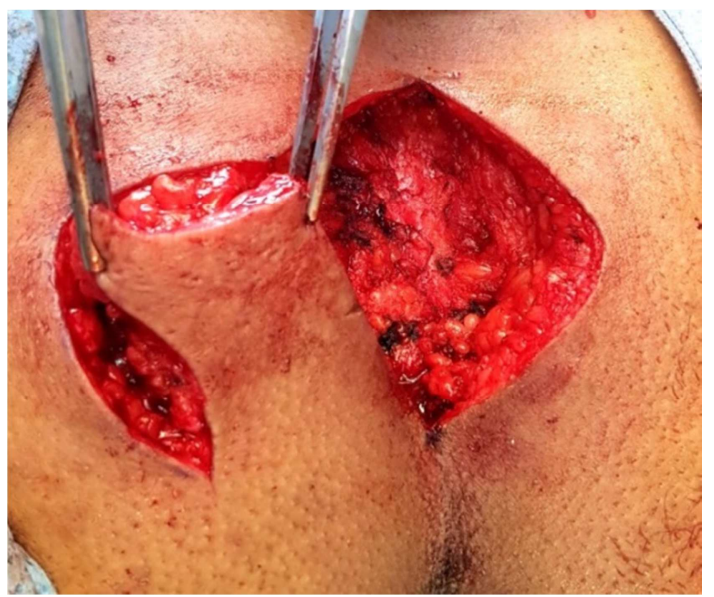

Figure 4. Showing complete excision of the rhomboid incision including the sinuses down to the sacral fascia and the flap completely mobilized and dissected down to the muscle and ready to be rotated medially to cover the defect.

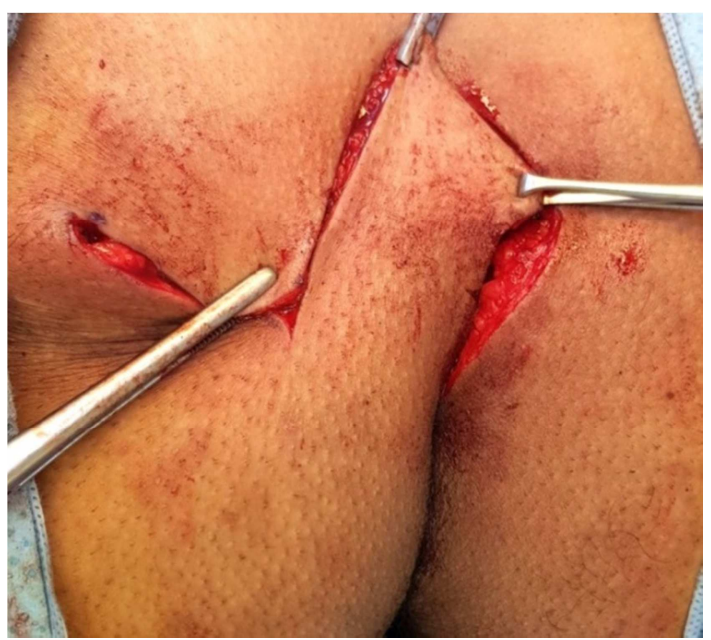

Figure 5. Showing rotation of the mobilized flap medially to cover the defect after excision of the rhomboid incision including the sinuses down to the sacral fascia. 


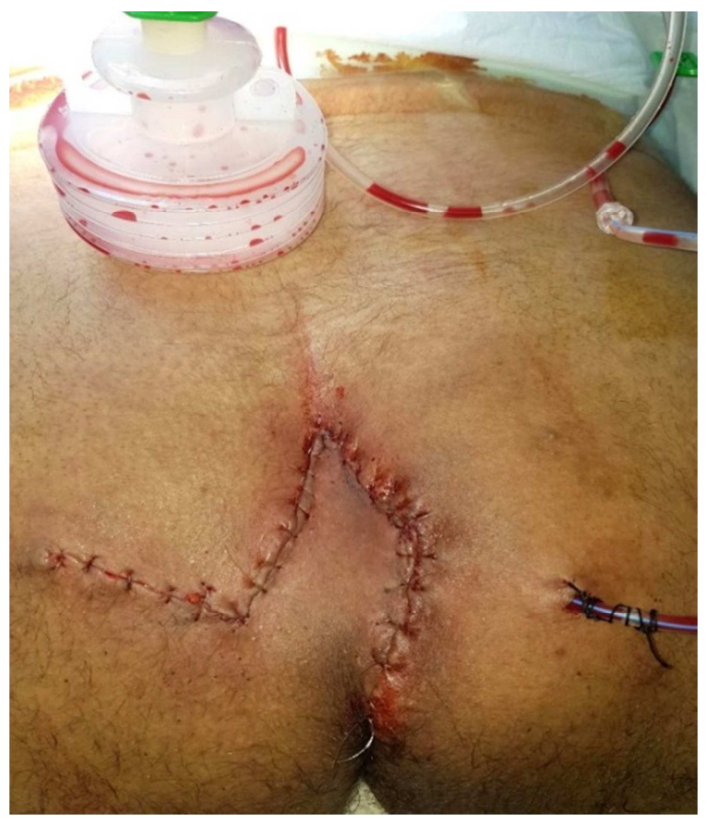

Figure 6. Showing wound closure with interrupted skin sutures with Monocryl 3 zero and suction drain no 16 extracted from separate stab wound away from the flap side.

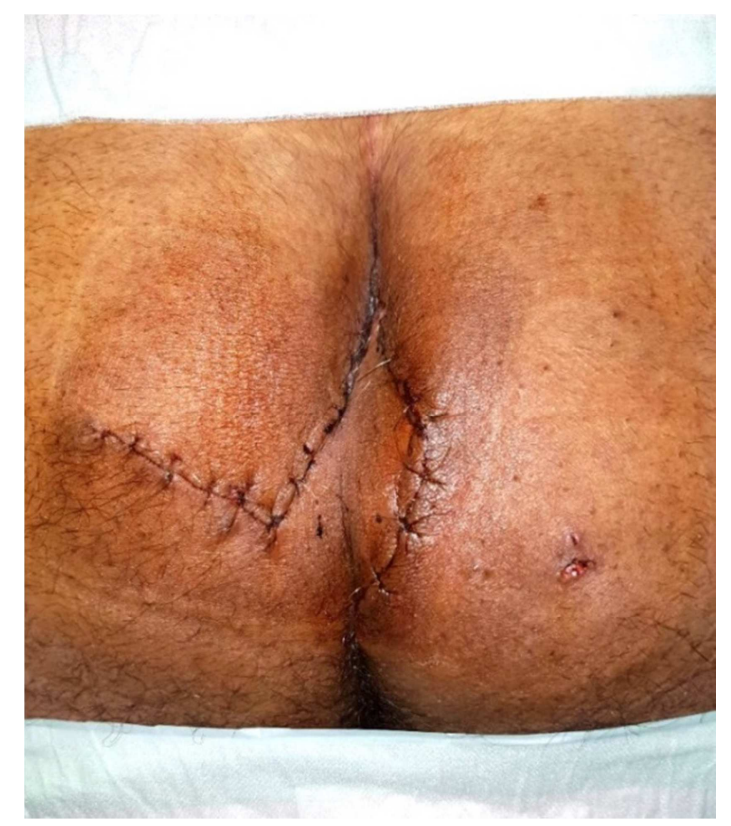

Figure 7. Showing good wound healing one week postoperative.

A primary closure is associated with faster wound healing (complete epithelialization) and a sooner return to work, but a delayed (open) closure is associated with a lower likelihood of pilonidal disease recurrence. This is best illustrated by a meta-analysis of 26 randomized trials including 2530 patients [4].

Closure of the wound is more cosmetically acceptable for some patients and is associated with a shorter healing time and less time off work. Wide local excision and primary closure have been advocated for the treatment of PS by some researchers, but the resulting scar remains in the midline and is associated with a high incidence of recurrence [10]. In order to solve the problem of midline scar formation and reduce the depth of the natal cleft, the Karydakis technique uses an eccentric elliptical incision for sinus excision and a flap is mobilized from the medial side of the wound, leaving the final suture line at either side of the midline.

Reconstruction of the defect with Limberg flap has many advantages as it is easy to perform and design, and it flattens the natal cleft with large vascularized pedicle, sutured without tension. This in turn maintains good hygiene, reducing the friction, preventing maceration, and avoiding scar in the midline. This flap procedure found better than simple excision and closure, marsupialization [11].

In this study, 30 patients with sacrococcygeal pilonidal sinus were managed with rhomboid excision and Limberg flap reconstruction. Recurrence was noted in one patient in the 6 months postoperative follow up which represented (3.33\%) from all patients. Akin et al operated on 411 patients and reported recurrence rates of $2.9 \%$ [12].

Return to work and normal activity in our study was 3 to 4 weeks, hospital stay 24 to $48 \mathrm{hrs}$. No cases of wound infection, only one case of seroma and 2 cases of small wound japing were recorded and all managed conservatively in about 2 weeks on daily wound dressing. Moreover, Boshnaq et al. [13] was clarified that Limberg flap presents a safe and effective method that can be offered for patients with primary or recurrent pilonidal sinus disease (PSD).

\section{Conclusion}

Limberg rhomboid flap in sacrococcygeal pilonidal sinus is useful in primary and recurrent pilonidal sinus, has been associated with lower complication rates, lower healing time, and recurrence rates. It easy to perform flattens the natal cleft with a large well-vascularized pedicle that can be sutured without tension, minimal postoperative pain, midline dead space and scar is avoided, and reduces hospital stay and time to resume normal activities.

Finally we are concluded that Limberg rhomboid flap reconstruction after excision of sacrococcygeal pilonidal sinus is meticulous, safe, easy to be done, low operative time, low post-operative pain, low hospital stays, early return of the patient to work, suitable to primary and recurrent cases with low local recurrent and meet acceptance from the patient preoperative during discussion with the patient for writing the surgical consent and postoperative due to the previous benefits.

\section{References}

[1] Hodges RM. (1880): Pilonidal sinus. Boston Med Surg J., 103:485-586.

[2] Khanna A, Rombeau JL (2011): Pilonidal disease. Clin Colon Rectal Surg., 24(1):46-53.

[3] Al-Hassan HK, Francis IM, Neglen P. (1990): Primary closure or secondary granulation after excision of pilonidal sinus. Acta Chir Scand., 156 (3):695-699. 
[4] Al-Khamis A, McCallum I, King PM, Bruce J. (2010): Healing by primary versus secondary intention after surgical treatment for pilonidal sinus. Cochrane Database Syst Rev. 20 (1): CD006213. doi: 10. 1002/14651858. CD006213. pub3.

[5] Ardelt M, Dittmar Y, Rauchfuss F, Fahrner R, Scheuerlein H, Settmacher U. (2015): Classic Limberg Flap Procedure for Treatment of a Sacrococcygeal Pilonidal Sinus Disease Explanation of the Surgical Technique. Zentralbl Chir., 140(5):473-5 doi: 10. 1055/s-0035-1557760. Epub 2015 Oct 20 .

[6] Abu Galala KH, Salam IM, Abu Samaan KR, El Ashaal YI, Chandran VP, Sabastian M, Sim AJ. (1999): Treatment of pilonidal sinus by primary closure with a transposed rhomboid flap compared with deep suturing: a prospective randomised clinical trial. Eur J Surg., 165(5):468-72.

[7] Akca T, Colak T, Ustunso B, Kanik A, Aydin S. (2005): Randomized clinical trial comparing primary closure with the Limberg flap in the treatment of primary sacrococcygeal pilonidal disease. Brit J Surg., 92:1081-1084. doe: 10. 1002/bjs. 5074.

[8] Mayo OH. (1833): Observations on injuries and diseases of the rectum, London: Burgess and Hill, London. p. 45-46.
[9] Classic articles in colonic and rectal surgery. Louis A. Buie, M. D. 1890-1975: Jeep disease (pilonidal disease of mechanized warfare). Dis Colon Rectum. 1982 May-Jun; 25(4):384-90.

[10] Muzi MG, Milito G, Cadeddu F, Nigro C, Andreoli F, Amabile D, Farinon AM. (2010): Randomized comparison of Limberg flap versus modified primary closure for the treatment of pilonidal disease. Am J Surg., 200(1):9-14. doi: 10. 1016/ j.amjsurg. 2009. 05. 036.

[11] Azab AS, Kamal MS, Saad RA, Abou al Atta KA, Ali NA. (1984): Radical cure of pilonidal sinus by a transposition rhomboid flap. Br J Surg., 71(2):154-5.

[12] Akin M, Gokbayir H, Kilic K, Topgul K, Ozdemir E, Ferahkose Z. (2008): Rhomboid excision and Limberg flap for managing pilonidal sinus: long-term results in 411 patients. Colorectal Dis., 10(9):945-8.

[13] Boshnaq M, Phan YC, Martini I, Harilingam M, Akhtar M, Tsavellas G (2018): Limberg flap in management of pilonidal sinus disease: systematic review and a local experience, Acta Chirurgica Belgica, 118:2, 78-84, DOI: 10.1080/00015458.2018.1430218. 